\section{AB0301 CRITERIA FULLFILLMENT OF PRIMARY SJÖGREN'S SYNDROME ACCORDING TO TIME OF EVOLUTION OF SICCA SYMPTOMS}

C. M. Gamboa-Alonso ${ }^{1}$, D. Vega-Morales ${ }^{1}$, J. C. Riegatorres ${ }^{1}$, B. R. Vázquez Fuentes ${ }^{1}$, L. Á. Ceseñas-Falcónn ${ }^{1}$, G. Figueroa-Parra' ${ }^{1}$ D. Á. Galarza-Delgado'. ${ }^{1}$ Universidad Autónoma de Nuevo León, Reumatología e Inmunología Clínica, Monterrey, Mexico

Background: Primary Sjögren's Syndrome (PSS) is an autoimmune disease characterized by sicca symptoms, autoantibodies and lymphocytic infiltrate in exocrine glands.

Classification and diagnosis of PSS is performed by applying 2002 AECG, 2012 ACR or 2016 ACR-EULAR criteria.

Time of evolution of symptoms has been associated with different clinical, serological and pathology findings.

Objectives: Document sicca profiles and compare findings according to time of evolution of symptoms.

Methods: A descriptive retrospective observational study was performed in 116 patients with sicca symptoms in a Rheumatology center in México.

Patients were separated according to time of evolution of sicca symptoms in $\leq 1$ year, $\leq 2$ years, $\leq 3$ years, and $\geq 3$ years. Clinical, serological, and histopathology features were assessed at each established time.

Time from symptom onset to diagnosis was reported in median and interquartil range (IQR); age at symptom onset and age at diagnosis was expressed in mean and standard deviation (SD).

Patients were classified as PSS if they fullfilled the 2002 AECG or 2016 ACR-EULAR criteria. Serology of Rheumatoid factor (RF), ANA, Anti-Ro, and Anti-La were documented.

Description of MSGB was performed reporting presence of foci, lobules, atrophy, adipose tissue infiltration and ductal dilatation.

Results: One hundred and sixteen sicca patients had a complete profile and 97 (83.62\%) fulfilled AECG 2002 SSP criteria. Eighty-two (70.6\%) patients with an ocular staining score were able to classify as PSS using the 2016 ACR-EULAR criteria.

Of the sicca cohort, $112(96.6 \%)$ were female. The mean age of symptom onset was 48.4 (SD 13.11) years, and the mean age at diagnosis was 53.33 (SD 12.43) years. The median time from symptom onset to diagnosis was 36 (IQR 12-84) months.

Fullfilment the 2002 AECG PSS classification criteria according to duration of sicca symptoms is described in Table 1.

Table 1. Classification PSS criteria according to time of evolution of sicca symptoms

$<12$ months $<24$ months $<36$ months $>37$ months

\begin{tabular}{|c|c|c|c|c|c|}
\hline Oral symptoms & $28 \%$ & $40 \%$ & $48 \%$ & $89 \%$ & \\
\hline Ocular symptoms & $26 \%$ & $39 \%$ & $47 \%$ & $93 \%$ & \\
\hline Altered Salivary flow rate test & $21 \%$ & $32 \%$ & $41 \%$ & $81 \%$ & \\
\hline Altered Schirmer test & $19 \%$ & $28 \%$ & $38 \%$ & $75 \%$ & \\
\hline Positive Anti Ro & $13 \%$ & $19 \%$ & $26 \%$ & $47 \%$ & \\
\hline Positive Anti La & $3 \%$ & $6 \%$ & $10 \%$ & $16 \%$ & \\
\hline$\geq 1$ foci score/4mm2 & $14 \%$ & $24 \%$ & $34 \%$ & $67 \%$ & \\
\hline 2002 AECG Criteria* & $22 \%$ & $34 \%$ & $44 \%$ & $84 \%$ & \\
\hline \multicolumn{6}{|l|}{ AECG-American European } \\
\hline & $0 \%$ & $25 \%$ & $50 \%$ & $75 \%$ & $100 \%$ \\
\hline
\end{tabular}

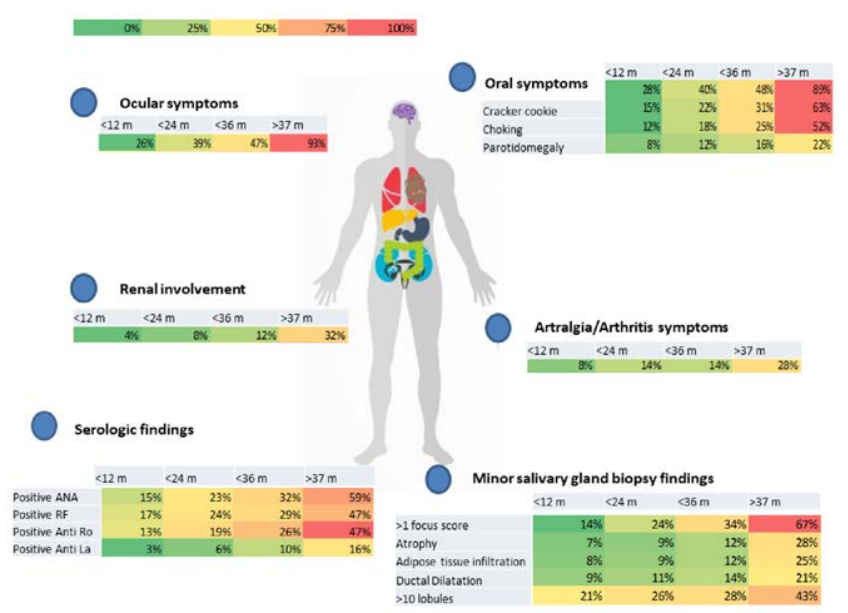

Figure 1. Clinical, serologic and histopathologic findings according to sicca symptom duration
Extra-classification criteria such as severe oral symptoms (choking, cracker cookie) were more common with prolonged symptom duration. Figure 1

Conclusion: The probability and capability of fulfilling PSS criteria is time dependent, this should be taken into consideration when evaluating patients referring sicca symptoms. Longer symptom duration was associated with more severe clinical, serological and histopathology profiles.

\section{REFERENCES:}

[1] Shiboski CH, Baer AN, Shiboski SC, et al (2018) Natural History and Predictors of Progression to Sjögren's Syndrome Among Participants of the Sjögren's International Collaborative Clinical Alliance Registry. Arthritis Care Res (Hoboken) 70:284-294. https://doi.org/10.1002/acr.23264

Acknowledgements: We thank Dr. Jazzia Emily Díaz Angulo and Dr. Gabriela Luna Limón for their help in data compilation.

Disclosure of Interests: None declared

DOI: 10.1136/annrheumdis-2021-eular.922

\section{AB0302 FACTORS ASSOCIATED WITH GESTATIONAL DIABETES MELLITUS (GDM) IN A MULTI-ETHNIC SYSTEMIC LUPUS ERYTHEMATOSUS (SLE) COHORT}

S. S. Shaharir ${ }^{1}$, R. Mustafar ${ }^{2}$, M. S. Mohamed Said ${ }^{1}$, R. Abd Rahman ${ }^{3}$ ${ }^{1}$ Universiti Kebangsaan Malaysia Medical Centre, Rheumatology Unit, Department of Medicine, Cheras, Malaysia; ${ }^{2}$ Universiti Kebangsaan Malaysia Medical Centre, Nephrology Unit, Department of Medicine, Cheras, Malaysia; ${ }^{3}$ Universiti Kebangsaan Malaysia Medical Centre, Obstetrics and Gynaecology, Cheras, Malaysia

Background: The risks of insulin resistance and diabetes mellitus are elevated in systemic lupus erythematosus (SLE) patients. The use of glucocorticoid and anti-double stranded DNA antibodies positive are among the factors reported to be associated with the risk of gestational diabetes mellitus (GDM) in SLE patients However, the relationship between GDM in Asian SLE patients is still obscure. Objectives: To determine the prevalence of gestational diabetes mellitus (GDM) in a multi-ethnic SLE cohort in Malaysia and the associated risk factors.

Methods: This was a retrospective study of SLE pregnant women who have completed their antenatal care in Universiti Kebangsaan Malaysia Medica Centre (UKMMC) from 2004 until 2019. Screening and diagnosis of gestational diabetes mellitus (GDM) were as recommended in the guidelines by the Ministry of Health Malaysia. Information on SLE disease activity and treatment at 6 months before pregnancy and during pregnancy were determined from the medical records. Univariate and multi-variable logistic regression analyses were performed to determine the factors associated with GDM in the SLE patients. Results: A total of 89 patients with 202 pregnancies were included in the study. Malay was the predominant ethnic in this cohort $(n=82,67.2 \%)$, followed by Chinese $(n=33,27.0 \%)$ and Indian $(n=7,5.7 \%)$. The most common system involvement of SLE was musculoskeletal $(n=91,74.6 \%)$, followed by haematological $(n=78,63.9 \%)$, lupus nephritis $(54.9 \%, n=67)$ and mucocutaneous $(n=66,54.1 \%)$. The prevalence of GDM was $8.9 \%(n=18)$. More patients with GDM had positive anti-cardiolipin IgG antibody (aCL IgG) and lupus anticoagulant (LA) antibody as compared to the patients with no GDM, (55.6\% vs $25.8 \%, \mathrm{p}=0.01)$ and $(50.0 \%$ vs $25.4 \%, \mathrm{p}=0.05)$ respectively. On the other hand, the use of hydroxychloroquine (HCQ) in pregnancy was significantly lower in GDM patients (11.1\%) as compared to no GDM group $(39.1 \%), p=0.02$. There was no significant difference in the ethnicity, SLE system involvement, disease activity status and immunosupressant use including steroid, azathioprine and cyclosporine $A$ at 6 months before and during pregnancy between the GDM and non-GDM group. A forward logistic regression which include $\mathrm{ACL} \lg \mathrm{G}$ $\mathrm{LA}$ and $\mathrm{HCQ}$ use in pregnancy, only the HCQ use remained significantly associated with lower risk of GDM in the model with $\mathrm{OR}=0.12,95 \% \mathrm{C} . \mathrm{I}=0.02-0.94, \mathrm{p}=0.04$. Conclusion: Our study demonstrates the potential benefit of hydroxychloroquine in reducing the risk of gestational diabetes mellitus in SLE patients. The prevalence of antiphospholipid antibodies particularly $\mathrm{aCL} \operatorname{IgG}$ and LA was found to be higher among patients with GDM. Further prospective studies are needed to confirm this association.

\section{REFERENCES:}

[1] Dong Y, Dai Z, Wang Z, et al. Risk of gestational diabetes mellitus in systemic lupus erythematosus pregnancy: a systematic review and meta-analysis. BMC Pregnancy and Childbirth. 2019 May;19(1):179. DOI: 10.1186/ s12884-019-2329-0.

Disclosure of Interests: None declared

DOI: 10.1136/annrheumdis-2021-eular.1044

\section{$\mathrm{AB} 0303$ \\ VALIDATION OF THE THREE CLASSIFICATION CRITERIA FOR SYSTEMIC LUPUS ERYTHEMATOSUS (SLE) ON A PATIENT COHORT FROM A V.A. NASONOVA SCIENTIFIC RESEARCH INSTITUTE OF RHEUMATOLOGY: A PROSPECTIVE STUDY}

T. Reshetnyak ${ }^{1,2}$, A. Shumilova ${ }^{3}$, S. Glukhova ${ }^{4}$, F. Cheldieva ${ }^{1,2}$, T. Lisitsyna ${ }^{1}$, N. Seredavkina ${ }^{1}$, A. Lila ${ }^{2,3} \cdot{ }^{1}$ V.A. Nasonova Research Institute of Rheumatology, 
Moscow, Russia; Vascular Rheumatology, Moscow, Russian Federation; ${ }^{2}$ Russian Medical Academy of Continuing Professional Education, Ministry of Health of Russia, Department of Rheumatology, Moscow, Russian Federation; ${ }^{3}$ V.A. Nasonova Research Institute of Rheumatology, Moscow, Russia; Department of Rheumatology, Moscow, Russian Federation; ${ }^{4}$ V.A. Nasonova Research Institute of Rheumatology, Moscow, Russia;, Laboratory of Medical and Social Problems of Rheumatology, Moscow, Russian Federation

Background: Classification criteria for SLE need validation and selection of the most advanced ones for clinical practice

Objectives: To validate Systemic Lupus International Collaborating Clinics (SLICC)-12, American College of Rheumatology (ACR)-97 and 2019 European League against Rheumatism/American College of Rheumatology (EULAR/ACR2019) for Systemic

Lupus Erythematosus classification criteria on a patient cohort from V.A. Nasonova Scientific Research Institute of Rheumatology (Moscow)

Methods: This prospective study included 252 ( $n=152$ with SLE and $n=100$ non SLE: 74 - with systemic sclerosis (SC) and 26 - with primary antiphospholipid syndrome (pAPS)) patients who were sequentially admitted to the 4th Rheumatology Department from December 2018 to June 2020. All of the patient records were reevaluated by expert rheumatologist in order to determine if they agree with the diagnosis. For every patient, a check-list of SLE-related features was filled out. The association between clinical diagnosis and diagnosis generated on the basis of ACR-97, SLICC-12 and EULAR/ACR-2019classification criteria was assessed. The overall sensitivity and specificity of ACR-97, SLICC-12 and EULAR/ACR-2019 classifications, as well as the sensitivity and specificity according to disease duration was calculated. The predictive value of every criterion in ACR-97, SLICC-12 and 2019 EULAR/ACR classification was assessed using logistic regression analysis and receiver operating characteristic (ROC) curves.

Results: According to ACR-97 criteria, reliable SLE was diagnosed in 131 (86\%), according to SLICC-2012 - in 145 (95.3\%) and according to EULAR/ACR-2019 - in $144(94.7 \%)$ of 152 patients, respectively. We identified the criteria that were significantly more represented in the SLE-group. The sensitivity and specificity (Table 1) did not change depending on the ANA titers (1/160 and 1/320) and the duration of the disease.

Table 1. The sensitivity and specificity of SLE criteria in 152 SLE patients and 100 non SLE patients

\begin{tabular}{lcccc}
\hline SLE classification criteria & AUC, 95\% Cl & Cut point & Sensivity & Specifity \\
\hline ACR-97 & $0.654(0.550-0.758)$ & 4 & 89.7 & 36 \\
SLICC-12 & $0.616(0.505-0.728)$ & 4 & 93.1 & 43 \\
2019 EULAR/ACR & $0.609(0.492-0.727)$ & $10^{*}$ & 97.4 & 48
\end{tabular}

Note. ACR-97 - American College of Rheumatology classification from 1997: SIICC-12 Systemic lupus International Collaboration Clinics classification from 2012; 2019 European League Against Rheumatism/American College of Rheumatology. ANA - anti-nuclear antibody more than 1/320 $n=116$; ANA $<320 n=36$. AUC - area under the ROC curve; Cut point - reference point for the number of criteria, ${ }^{*}$ number of points $\geq 10$.

When excluding patients with SC in a single-factor logistic model of patients with SLE and pAPS, indicating the number of criteria, the sensitivity was for ACR-97 - 86, for SIICC-12 - 95, for 2019 EULAR/ACR - 95, the specificity was 100, 62 and 62 , respectively.

Conclusion: Evaluation of the criteria by level ANA revealed the highest sensitivity for 2019 EULAR/ACR and SLICC-12, the specificity was low for all three criteria. In the analysis of patients with SLE and pAPS, the sensitivity was highest for 2019 EULAR/ACR and SLICC-12, the specificity was 100 for ACR-97.

Disclosure of Interests: None declared

DOI: 10.1136/annrheumdis-2021-eular. 1051

\section{AB0304 LEVELS AND TRENDS IN PREMATURE MORTALITY BURDEN DUE TO SYSTEMIC LUPUS ERYTHEMATOSUS IN CUBA}

H. Hernández-Negrín ${ }^{1}{ }^{1}$ Universidad de Ciencias Médicas de Villa Clara Internal Medicine, Santa Clara, Cuba

Background: Analysis of the cause of premature mortality is an essential function of public health surveillance. A variety of methods have been used to accurately assess and report the level and trends of premature mortality; however, many have significant limitations, particularly in capturing actual early deaths. Years of life lost (YLL) has been recognized as a robust and comprehensive measure of premature mortality. ${ }^{1}$ Systemic lupus erythematosus (SLE) is among the leading causes of premature mortality burden in young women in the United States and premature mortality from SLE is increasing in Latin America. ${ }^{2,3} \mathrm{How}-$ ever, the premature mortality burden from SLE has not been fully characterized in Cuba.
Objectives: To identify levels and trends in premature mortality burden due to SLE in Cuba during 2001-2018.

Methods: Data were obtained from the mortality database of the Ministry of Public Health of Cuba (International Classification of Diseases-10, code 32 as underlying cause of death). YLL were calculated using the standard life expectancy of the WHO's global health estimates. Age- and sex-specific YLL rates were calculated using the Cuban mid-year population estimates and the age-standardized YLL rate (ASYR) was estimated by the direct method using the WHO's standard population. The 2001-2018 temporal trends were analyzed using Jointpoint software.

Results: During 2001-2018, 1475 patients died from SLE. These deaths contributed 66605 YLL (women= 59 166; men=7 433), with a mean of 45.1 YLL per death (women= 45.2; men=44.5). The 40-44 age group was the most affected in both sexes with 9266 YLL (women=8 177; men=1 086). The ASYR was higher in 2018 than in 2001 for both sexes (Figure 1). A significantly increasing trend in ASYR due to SLE was identified throughout the period (average annual percent change $[\mathrm{AAPC}]=1.9 ; 95 \%$ confidence interval $[\mathrm{Cl}]=0.9$ to 3.0 ), more pronounced in men $(A A P C=7.0 ; 95 \% \mathrm{Cl}=3.5$ to 10.6) than in women $(\mathrm{AAPC}=1.3$ $95 \% \mathrm{Cl}=0.3$ to 2.3 ).

Conclusion: The high levels and growing trends in premature mortality burden from SLE in Cuba, demands its recognition as an important health problem and immediate actions that help mitigate it.

\section{REFERENCES:}

[1] Martinez R, Soliz P, Caixeta R, Ordunez P. Reflection on modern methods: years of life lost due to premature mortality-a versatile and comprehensive measure for monitoring non-communicable disease mortality. Int $\mathrm{J}$ Epidemiol. 2019; 48(4): 1367-1376.

[2] Yen EY, Singh RR. Lupus - an unrecognized leading cause of death in young women: Population-based study using nationwide death certificates, 2000 2015. Arthritis Rheumatol. 2018;70:1251-5.

[3] Soares CR, Viana K, Moraes-Santos F, Vieira CL, Lamarão F, Iglesias-Rodriguez $M$. Mortality rate and premature mortality due to systemic erythematosus lupus in Latin America US and England and Wales. Value Health. 2015;18:A805-81.

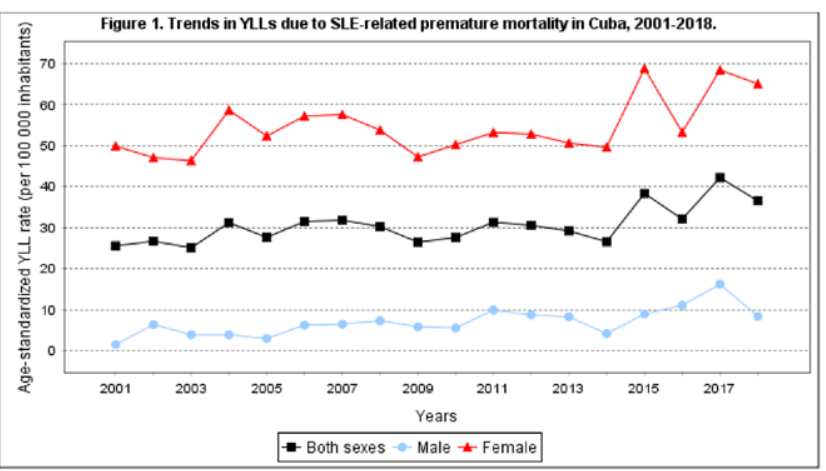

Disclosure of Interests: None declared

DOI: 10.1136/annrheumdis-2021-eular.1147

\section{$\mathrm{AB} 0305$}

IS THERE HYPERCOAGULATION IN PATIENTS WITH ANTIPHOSPHOLIPID SYNDROME AND BEHCET'S DISEASE?

N. Seredavkina ${ }^{1}$, T. Reshetnyak ${ }^{1,2}$, T. Lisitsyna ${ }^{1}$, A. Lila ${ }^{1,2} .{ }^{1}$ VA Nasonova Research Institute of Rheumatology, Department of Vascular Rheumatology, Moscow, Russian Federation; ${ }^{2}$ Federal State Budgetary Educational Institution of Further Professional Education "Russian Medical Academy of Continuous Professional Education" of the Ministry of Healthcare of the Russian Federation, Department of Rheumatology, Moscow, Russian Federation

Background: Whereas antiphospholipid syndrome (APS) is a non-inflammatory vasculopathy and is associated with thrombosis in $98 \%$ of cases, Behcet's disease (BD) is a systemic vasculitis of unknown etiology, characterized by vascular damage of any calibre. Both venous and arterial thromboses occur in $45 \%$ of BD patients and are associated not with hypercoagulable disease but with inflammatory changes in the vascular wall mediated by hypersecretion of pro-inflammatory cytokines and endothelial cells dysfunction. Thrombodynamics (TD) is a new global test for diagnosing plasma haemostasis disorders, identifying bleeding and thrombosis risks, and can be used to detect a prothrombotic state and assess the influence of disease activity and course on the hypercoagulation process.

Objectives: comparative assessment of TD in patients with APS and BD before anticoagulant therapy (AC)

Methods: The study included 20 patients ( 9 APS and $11 \mathrm{BD}$ ) and 8 age and sexmatched healthy controls $(\mathrm{HC})$. None of the subjects received AC. Thromboses 